\title{
Spectral Models of Convection\#dominated Accretion Flows
}

\section{Citation}

Ball, Gregory H., Ramesh Narayan, and Eliot Quataert. 2001. “Spectral Models of Convection\#dominated Accretion Flows." The Astrophysical Journal 552 (1): 221-26. https:// doi.org/10.1086/320465.

\section{Permanent link}

http://nrs.harvard.edu/urn-3:HUL.InstRepos:41384938

\section{Terms of Use}

This article was downloaded from Harvard University's DASH repository, and is made available under the terms and conditions applicable to Other Posted Material, as set forth at http:// nrs.harvard.edu/urn-3:HUL.InstRepos:dash.current.terms-of-use\#LAA

\section{Share Your Story}

The Harvard community has made this article openly available.

Please share how this access benefits you. Submit a story.

Accessibility 


\title{
Spectral Models of Convection-Dominated Accretion Flows
}

\author{
Gregory H. Ball, Ramesh Narayan \\ Harvard-Smithsonian Center for Astrophysics \\ 60 Garden Street, Cambridge MA 02138, U.S.A \\ and \\ Eliot Quataert \\ Institute for Advanced Study, Einstein Drive, Princeton, NJ 08540
}

\begin{abstract}
For small values of the dimensionless viscosity parameter, namely $\alpha \lesssim 0.1$, the dynamics of non-radiating accretion flows is dominated by convection; convection strongly suppresses the accretion of matter onto the central object and transports a luminosity $\sim 10^{-3}-10^{-2} \dot{M} c^{2}$ from small to large radii in the flow. A fraction of this convective luminosity is likely to be radiated at large radii via thermal bremsstrahlung emission. We show that this leads to a correlation between the frequency of maximal bremsstrahlung emission and the luminosity of the source, $\nu_{\text {peak }} \propto L^{2 / 3}$. Accreting black holes with X-ray luminosities $10^{-4} L_{E d d} \gtrsim L_{X}(0.5-10 \mathrm{keV}) \gtrsim 10^{-7} L_{E d d}$ are expected to have hard X-ray spectra, with photon indices $\Gamma \sim 2$, and sources with $L_{X} \lesssim 10^{-9} L_{E d d}$ are expected to have soft spectra, with $\Gamma \sim 3.5$. This is testable with Chandra and XMM.
\end{abstract}

\section{Introduction}

At luminosities less than a few per cent of the Eddington luminosity, black holes can accrete via an advection-dominated accretion flow, or ADAF (Ichimaru 1977; Rees et al. 1982; Narayan \& Yi 1994, 1995; Abramowicz et al. 1995; see Kato, Fukue \& Mineshige 1998 and Narayan, Mahadevan \& Quataert 1999 for reviews). Analytical calculations have shown that such flows should be convectively unstable (Narayan \& Yi 1994, 1995; see also Begelman \& Meier 1982); the instability has been confirmed in numerical simulations (Igumenshchev, Chen \& Abramowicz 1996; Igumenshchev \& Abramowicz 1999, 2000; Stone, Pringle \& Begelman 1999; Igumenshchev, Abramowicz \& Narayan 2000).

Igumenshchev \& Abramowicz $(1999,2000)$ found that convection is strong whenever the viscosity parameter $\alpha$ is small, roughly $\alpha \lesssim 0.1$. Such "convection-dominated accretion flows," or CDAFs, have a very different structure than ADAFs (Stone et al. 1999). The density of the accreting gas varies as $\rho \propto R^{-1 / 2}$ (where $R$ is the radius) rather than $\rho \propto R^{-3 / 2}$, and the mean radial velocity varies as $v \propto R^{-3 / 2}$ rather than $v \propto R^{-1 / 2}$ (Narayan, Igumenshchev \& Abramowicz 2000, hereafter NIA).

NIA showed that, in the numerical simulations, the Reynolds stress due to convection is negative, which implies that convection moves angular momentum inwards rather than outwards. This property of convection was discussed in the context of thin accretion disks by Ryu \& Goodman (1992) and Stone \& Balbus (1996). In the case of a CDAF, convection is so strong that the angular momentum it transports inwards nearly cancels the normal outward transport by viscosity (NIA; Quataert \& Gruzinov 2000). As a result, one has a nearly static accretion flow in which most of the gas circulates in convective eddies rather than accreting onto the central object. For fixed boundary conditions at large radii the mass accretion rate in a CDAF is thus much smaller than in a non-convecting ADAF or a Bondi flow. 
In this paper we highlight observational consequences of CDAF models, focusing in particular on spectra. Some of our results are similar to those of Di Matteo et al. (1999, 2000; DM) and Quataert \& Narayan (1999a; QN) who followed up the proposal of Blandford \& Begelman (1999; see also Narayan \& Yi 1994, 1995) that a significant fraction of the mass in an ADAF would be lost to an outflow/wind, rather than accreting onto the central object. The importance of outflows can be parametrized by a radial density profile, $\rho \propto R^{-3 / 2+p}$, with $p \epsilon[0,1]$. The density profile in a CDAF is equivalent to $p=1$. Thus DM's and QN's spectral models, which considered various values of $p$, capture many of the features of the CDAF spectra calculated here; this is discussed in more detail in $\S 4$.

Convection in CDAFs transports a luminosity $L_{c} \sim 10^{-3}-10^{-2} \dot{M} c^{2}$ from small to large radii; the energy is supplied by the small amount of mass accreting onto the black hole. What happens to this energy at large radii? NIA and Igumenshchev \& Abramowicz (2000) suggested that some, perhaps most, of the energy might be radiated from the outer regions of the CDAF as thermal bremsstrahlung emission. We present model spectra for such flows.

In the next section we present analytical expressions for the luminosity and bremsstrahlung spectrum of CDAFs ( $(2)$. We then show more detailed numerical calculations ( 33.1$)$ and compare CDAF models with ADAF models (§3.2). In $\S 4$ we summarize and emphasize some implications of our analysis.

\section{Self-Similar Scalings for Bremsstrahlung Luminosity and Spectrum}

In this section we use the self-similar CDAF solution described by Narayan et al. (2000) and Quataert $\&$ Gruzinov (2000). We employ Schwarzschild units for the radius, i.e. $r=R / R_{\mathrm{S}}$, where $R_{\mathrm{S}}=2 G M / c^{2}=$ $2.95 \times 10^{5} \mathrm{~m} \mathrm{~cm}$ and $m$ is the black hole mass in solar units. We assume that the accretion flow extends from an outer radius $r_{\text {out }}$ down to an inner radius $r_{\text {in }}=1$ (to model a Schwarzschild black hole). The sound speed of a CDAF is nearly virial, $c_{s}^{2} \approx 0.37 c^{2} / r$, and the temperature is $T \equiv T_{0} / r \approx 10^{12} \mathrm{~K} / r$; the vertical scale height is thus of order the radius, $H \approx 0.6 R$.

The density of gas in a CDAF is given by

$$
\rho=\rho_{0} r^{-1 / 2}=\rho_{\text {out }}\left(\frac{r}{r_{\text {out }}}\right)^{-1 / 2}, \quad \rho_{0}=\rho_{\text {out }} r_{\text {out }}^{1 / 2} .
$$

We assume that the CDAF is in steady state with a constant mass accretion rate $\dot{M}$. Since $\rho$ varies as $r^{-1 / 2}$, the radial velocity must scale as $v \propto r^{-3 / 2}$. We normalize the velocity such that it is equal to $c$ at $r_{\text {in }}$.

As noted in $\S 1$, convection in CDAFs transports energy from small to large radii. We write the convective luminosity as $L_{c} \equiv \epsilon_{c} \dot{M} c^{2}$, where $\epsilon_{c} \sim 10^{-2}-10^{-3}$ is the convective "efficiency." Let a fraction $\eta_{c}$ of this energy be radiated at large radii by the gas in the CDAF; this radiation comes out as thermal bremsstrahlung emission. The bremsstrahlung luminosity of the CDAF, $L_{\mathrm{CDAF}}$, then satisfies

$$
L_{\mathrm{CDAF}}=\eta_{c} L_{\mathrm{c}}=\eta_{c} \epsilon_{c} \dot{M} c^{2} .
$$

For a given $r_{\text {out }}$, this relation fixes the value of $\rho_{\text {out }}$ and thus $\dot{M}$ (see below). Equivalently, for a given $\dot{M}$, equation (2) uniquely fixes the outer radius of the CDAF, $r_{\text {out }}$. It also implies an observationally interesting correlation between the luminosity and X-ray spectrum of a source.

The simplest possibility we can consider is that $\eta_{c}=1$, which corresponds to all the convected energy being radiated by the CDAF. This may apply to those objects in which the CDAF is formed by the "evaporation" of a thin accretion disk, as has been discussed in the context of ADAFs by several authors (e.g., Meyer 
\& Meyer-Hofmeister 1994; Narayan, McClintock, \& Yi 1996; Honma 1996; Meyer, Liu, \& Meyer-Hofmeister 2000). If a CDAF is surrounded by a thin disk, and if $\eta_{c}<1$, the convective luminosity impinging on the inner edge of the thin disk would be very much greater than that needed to unbind the disk material. One could imagine that $L_{c}$ would then "eat away" the inner edge of the disk until the condition $L_{\mathrm{CDAF}} \approx L_{c}$ is roughly satisfied. This condition is not guaranteed, however, since another possibility is that $\eta_{c}<1$ and the excess convective energy is used to eject a substantial fraction of the mass evaporated from the disk.

When there is no outer disk, it is unlikely that $\eta_{c}$ will be close to unity. For example, if a black hole accretes spherically from the ISM of a galaxy, as is believed to occur in, e.g., Sgr A* at the center of our Galaxy (e.g., Melia 1992; Narayan, Yi, \& Mahadevan 1995) or elliptical galaxies at the centers of cooling flows (Fabian \& Rees 1995; Mahadevan 1997; DM; Quataert \& Narayan 1999b), both $r_{\text {out }}$ and $\rho_{\text {out }}$ are determined by the properties of the host galaxy. It would then require considerable fine-tuning for $L_{C D A F}$ to be equal to $L_{c}$. It is more likely that we will have $L_{\mathrm{CDAF}}<L_{c}$, i.e $\eta_{c}<1$. The unradiated energy $\left(L_{c}-L_{C D A F}\right)$ will be transported out, heating the external medium and perhaps driving some of the gas away in an outflow.

The bremsstrahlung luminosity of a CDAF is given by

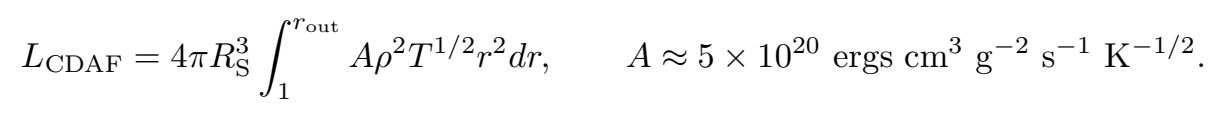

Performing the integral and equating $L_{\mathrm{CDAF}}$ to the convective luminosity, we may solve for $\rho_{0}$ :

$$
\rho_{0} \approx \frac{\eta_{c} \epsilon_{\mathrm{c}} c^{3}}{2 T_{0}^{1 / 2} R_{\mathrm{S}} A} r_{\text {out }}^{-3 / 2} \approx \frac{10^{-3}}{m r_{\text {out }}^{3 / 2}}\left(\frac{\eta_{c} \epsilon_{\mathrm{c}}}{10^{-2}}\right) \mathrm{g} \mathrm{cm}^{-3}
$$

Substituting this back into the expression for $L_{\mathrm{CDAF}}$ we find that the bremsstrahlung luminosity of the CDAF satisfies

$$
l_{\mathrm{CDAF}} \equiv \frac{L_{\mathrm{CDAF}}}{L_{\mathrm{Edd}}} \approx r_{\mathrm{out}}^{-3 / 2}\left(\frac{\eta_{c} \epsilon_{c}}{10^{-2}}\right)^{2},
$$

where $L_{\mathrm{Edd}}=1.25 \times 10^{38} \mathrm{~m} \mathrm{erg} \mathrm{s}^{-1}$. For $r_{\text {out }} \gg 1$, we see that the luminosity of a CDAF is $\ll L_{\mathrm{Edd}}$. Note that equation (5) can also be expressed as a relationship between the accretion rate and outer radius of the CDAF:

$$
\dot{m} \equiv \frac{\dot{M}}{\dot{M}_{\mathrm{Edd}}} \approx 10 r_{\text {out }}^{-3 / 2}\left(\frac{\eta_{c} \epsilon_{c}}{10^{-2}}\right),
$$

where $\dot{M}_{\text {Edd }}=L_{\text {Edd }} / 0.1 c^{2} \approx 10^{18} \mathrm{~m} \mathrm{~g} \mathrm{~s}^{-1}$ is the Eddington accretion rate.

A straightforward calculation (ignoring the frequency dependence of the Gaunt factor) shows that the bremsstrahlung spectrum from a CDAF consists of three parts:

$$
\begin{aligned}
\nu L_{\nu} & \propto \nu, \quad h \nu \ll k T_{\min }, \\
& \propto \nu^{-3 / 2}, \quad k T_{\min } \ll h v \ll k T_{\max }, \\
& \propto \exp \left(-h \nu / k T_{\max }\right), \quad k T_{\max } \ll h \nu,
\end{aligned}
$$

where $T_{\min }=T\left(r_{\text {out }}\right)$ is the minimum electron temperature in the flow, obtained at the outer radius, and $T_{\max }$ is the maximal electron temperature, obtained close to the black hole. If we include the Gaunt factor, the slope is shallower than unity for $h \nu \ll k T_{\min }$. 
The peak of the bremsstrahlung spectrum occurs at

$$
h \nu_{\text {peak }} \sim k T_{\min }=\frac{k T_{0}}{r_{\text {out }}} .
$$

Thus the position of the spectral peak depends on the outer radius. Since the luminosity of the CDAF is also related to the outer radius (eq. 5), we have the following relation between the luminosity and the location of the peak in the bremsstrahlung spectrum:

$$
h \nu_{\text {peak }} \approx 10\left(\frac{l_{\mathrm{CDAF}}}{10^{-6}}\right)^{2 / 3}\left(\frac{\eta_{c} \epsilon_{c}}{10^{-2}}\right)^{-4 / 3} \mathrm{keV}
$$

\section{Detailed Spectra}

The detailed spectra presented in this section were computed using a global dynamical model which includes a sonic transition close to the black hole (Quataert, in preparation). The model corresponds to a viscosity parameter $\alpha=0.03$ and has $\epsilon_{c}=0.0045$. The profiles of density and temperature in this model are quite close to the self-similar form assumed in the previous section.

In addition to bremsstrahlung emission, the spectral calculations presented here include synchrotron radiation and Compton scattering, assuming thermal electrons. These processes have been included by the methods described in Narayan, Barret \& McClintock (1997). Cooling from atomic processes has also been included, which can be important for $T<10^{8} \mathrm{~K}$ (i.e $r>10^{4}$ ).

In each model, the electron temperature $T_{\mathrm{e}}$ was computed self-consistently at each radius. Specifically, we solved an energy equation as a function of $r$ and ensured that the heating, cooling and energy advection of the electrons are in balance. For the heating, we assumed that a fraction $\delta$ of the viscous dissipation goes directly into the electrons.

\subsection{Models with a Pure CDAF}

Figure 1 shows model spectra corresponding to a CDAF around a $10^{8} M_{\odot}$ black hole. All the models shown have $\eta_{c}=1$ and either $\delta=0.01$ or $\delta=0.5$. The various curves correspond to different values of the outer radius, from $r_{\text {out }}=10^{6}$ down to 300 ; the corresponding values of $\dot{m}$ are given in the caption to the figure. (The precise values of $\dot{m}$ depend on details such as the values of $\alpha$ and $\epsilon_{c}$, and the global model of the gas dynamics, and probably should not be taken too literally. The trends are, however, likely to be robust.)

The main features discussed in $\S 2$ are clearly seen in Fig. 1. Consider first the models with $\delta=0.01$,

shown by dashed lines. We see that the X-ray spectrum is dominated by bremsstrahlung emission and consists of two distinct power-law segments on either side of a peak. The location of the peak is highly correlated with the luminosity, and follows the scaling given in equation (10).

At low frequencies, in the radio/submm band, there is a weak secondary peak in the spectrum which arises from synchrotron emission by the hot electrons. (This emission was computed assuming that the magnetic pressure in the plasma is a tenth that of the gas, cf. Quataert \& Narayan 1999c.) The synchrotron peak in the models is much weaker than in corresponding ADAF models (Narayan, Mahadevan \& Quataert 1997), where the synchrotron emission is often stronger than the bremsstrahlung emission. This is because the synchrotron emission comes from relativistic electrons close to the black hole, whereas the bremsstrahlung 
emission is from large radii. For a given outer density (and hence bremsstrahlung emission), the electron density at small radii in a CDAF is much smaller than that in an ADAF, causing the synchrotron emission in the CDAF to be strongly suppressed. This point was made by DM and QN in the context of winds from ADAFs, which also cause a reduction in the central density.

For higher values of $\dot{M}$ (or luminosity), Compton-scattering of synchrotron photons becomes important; this fills in the valley between the synchrotron and bremsstrahlung peaks.

The ions in the above models are close to virial: $T_{i} \sim 10^{12} \mathrm{~K}$ near the black hole. The electrons are comparatively cool, with a maximum temperature $T_{e, \max }<10^{10} \mathrm{~K}$. Models with $\delta=0.5$ (solid lines in Fig. 1) have much stronger electron heating and therefore significantly hotter electrons: $T_{e, \max } \sim 10^{11} \mathrm{~K}$. This causes the synchrotron peak to become much more pronounced compared to the $\delta=0.01$ models, though the emission is still less than in an equivalent ADAF model. The increased synchrotron emission and the hotter electrons both lead to an enhanced contribution from Compton scattering. Nevertheless, the peak of the spectrum is still dominated by bremsstrahlung emission, and so the scaling of the position of the peak with luminosity survives.

Figure 2 shows spectra for $\delta=0.01$ and $\delta=0.5$ for several values of the convective efficiency, $\eta_{c}$ : the two sets of models correspond to $\dot{m}=10^{-4}$ (solid lines) and $10^{-6}$ (dashed lines). In all the model sequences the synchrotron emission in the radio is relatively unaffected by changes in $\eta_{c}$; this is because the synchrotron radiation arises primarily at small radii and depends only on $\dot{m}$ and $\delta$. For the large $\dot{m}$ model, varying $\eta_{c}$ also has little effect on the X-ray spectrum. This is because most of the X-ray emission arises from small radii via inverse Compton scattering, rather than from large radii via bremsstrahlung. By contrast, for smaller $\dot{m}$ (and/or smaller $\delta$ ), inverse Compton emission is less important. The X-ray emission is then dominated by bremsstrahlung, which decreases with decreasing $\eta_{c}$.

Figure 3 shows the predicted X-ray photon index $\Gamma$ as a function of X-ray luminosity in Eddington units for $\delta=0.5$. Four sets of models are shown: $\eta_{c}=1,0.5,0.2,0.1$. The $2-5 \mathrm{keV}$ spectral indices were calculated by comparing the model luminosities at the two ends of the range, $2 \mathrm{keV}$ and $5 \mathrm{keV}$, respectively. Note the large increase of $\Gamma$ with decreasing luminosity. This arises because the peak in the spectrum moves to lower energies with decreasing luminosity (cf. eq. 10). Because the value of $\eta_{c}$ is unknown, there is some uncertainty regarding the value of the luminosity at which the transition in the value of $\Gamma$ occurs . For the range of $\eta_{c}$ considered here, covering an order of magnitude, we find that for $L_{X} / L_{E d d} \gtrsim 10^{-7}$ the X-ray spectrum is hard, with a photon index $\Gamma \sim 2$, while for luminosities $L_{X} / L_{E d d} \lesssim 10^{-9}$ the spectrum is significantly softer, with $\Gamma \sim 3.5$.

\subsection{CDAF vs. ADAF}

Figure 4 shows a comparison of CDAF and ADAF spectral models for $\delta=0.01$ and $\delta=0.5$. The accretion rates in the models are adjusted so that the $1 \mathrm{keV}$ X-ray luminosities are equal to either $10^{37}$ or $10^{40} \mathrm{erg} \mathrm{s}^{-1}$. In order to uniquely fix the models, we set $\eta_{c}=1$ in the CDAF models and choose the outer radii of the ADAF modles so that local viscous dissipation balances radiative cooling at this radius $(f \lesssim 1 / 2$ in the notation of Narayan \& Yi 1994).

For small $\delta$, Figure 4 shows that the CDAF and ADAF spectra are quite different. The X-ray emission in the CDAF is dominated by bremsstrahlung, while the emission in the ADAF is dominated by inverse Compton emission. In addition, the CDAF has a much lower synchrotron flux in the radio, because the 
density of gas close to the black hole is much smaller.

For very low luminosities $\left(<10^{-8} L_{E d d}\right)$, the difference between the CDAF and ADAF models at large $\delta$ is similar to that at small $\delta$. By contrast, for luminosities $>10^{-6} L_{E d d}$ the CDAF and ADAF spectra are very similar if $\delta$ is large. This is because the entire spectrum is produced over a small range of radii close to the black hole. Fixing the $1 \mathrm{keV} \mathrm{X}$-ray luminosity determines the properties of the flow in this region and thus the entire spectrum is rather similar.

\section{Discussion}

The main results of this paper are given in equations (6)-(10) and in the Figures. Equation (10) shows that a CDAF has a peak in $\nu L_{\nu}$ in the $\mathrm{X}$-ray band, whose position is correlated with the Eddington-scaled luminosity. Correspondingly, the spectral index of the X-ray spectrum varies with luminosity. At high luminosities, the peak in the spectrum is at higher energies than the typical band in which observations are carried out (few $\mathrm{keV}$ ), and the spectrum is hard with a photon index $\Gamma$ of order 2 (Fig. 3). At lower luminosities, the peak shifts to lower energies, and the observed band would correspond to energies above the peak. The spectrum then becomes very soft, with $\Gamma$ taking values of order 3.5.

There is some uncertainty in the quantitative details predicted here because the values of two parameters are not well determined. The parameter $\epsilon_{c}$ measures the fraction of the rest mass energy of the accreting gas that is carried outward by convection: $L_{c}=\epsilon_{c} \dot{M} c^{2}$. Our calculations correspond to $\epsilon_{c}=0.0045$, as determined from a global CDAF model with viscosity parameter $\alpha=0.03$ (Quataert, in preparation). Other values of $\alpha$ would give other estimates of $\epsilon_{c}$, though the uncertainty in $\epsilon_{c}$ is probably no more than a factor of a few either way.

A more serious uncertainty is in the parameter $\eta_{c}$, which measures the fraction of the convected luminosity $L_{c}$ that is radiated by the CDAF at large radii as thermal bremsstrahlung emission: $L_{C D A F}=$ $\eta_{c} L_{c}=\eta_{c} \epsilon_{c} \dot{M} c^{2}$. Figure 2 shows results spanning a range of $\eta_{c}$ from 0.01 to 1 . If $\eta_{c}$ is as small as 0.01 or even smaller, an accreting black hole would have a very small X-ray luminosity from thermal bremsstrahlung emission and would be extremely difficult to observe. This might explain highly underluminous galactic nuclei such as that in our own Galaxy. For models with low $\eta_{c}$, a relevant question is what happens to the part of the energy convected outwards that is not radiated, $\left(\left[1-\eta_{c}\right] L_{c} \approx L_{c}\right)$. If this energy is transported out to an external medium and radiated there, that contribution may dominate the observed spectrum (assuming the beam of the telescope is larger than the size of the emitting region). The luminosity of the source would then be larger than we have predicted and the spectrum would be softer.

The spectral models of CDAFs presented here are qualitatively similar to the ADIOS (ADAF + wind) models presented by DM and QN. The relative prominence of bremsstrahlung emission in the X-ray band and the suppression of synchrotron emission in the radio (as suggested by observations; see DM) are characteristic of density profiles flatter than the ADAF scaling of $\rho \propto r^{-3 / 2}$ (be they CDAF or ADIOS). In fact, the qualitative similarity of ADIOS and CDAF spectra implies that direct signatures of outflowing gas (and measrements of outflow mass rates) would be needed to confirm the ADIOS model. The primary difference between the CDAF and ADIOS models is that we have a unique radial density profile $\left(\rho \propto r^{-1 / 2}\right)$ in a CDAF, instead of a family of models $\left(\rho \propto r^{-3 / 2+p}\right.$ with $\left.0<p<1\right)$ in an ADIOS; also, the energy which powers bremsstrahlung emission at large radii is provided by convective transport from small radii in a CDAF, rather than by local viscous dissipation in an ADIOS. 
All the models presented in this paper correspond to a two-temperature plasma. For the models with $\delta=0.5$, however, the temperatures of the ions and electrons are similar, differing by less than a factor of 10. We have computed models with a one-temperature plasma, assuming that some plasma process other than Coulomb collisions rapidly equilibrates the ion and electron temperatures (cf Begelman \& Chiueh 1988). The results are not very different from those obtained with two-temperature models with $\delta=0.5$. Comptonization does, however, become more important relative to bremsstrahlung, especially for low values of $\eta_{c}$. In addition, the synchrotron emission in the radio is somewhat more prominent.

An important assumption of our analysis is that the electrons are thermal. Because of the low density in a CDAF, thermalization through Coulomb collisions and synchrotron self-absorption is virtually non-existent (cf. Mahadevan \& Quataert 1997); it is therefore possible to retain a power-law distribution of electrons. Moreover, such nonthermal acceleration is expected in the collisionless magnetized plasmas present in CDAFs. A power law tail of electrons would significantly modify the predicted synchrotron spectrum (e.g., Mahadevan 1998; Özel, Psaltis, \& Narayan 2000). In addition, the power-law electrons could contribute to X-ray emission through either synchrotron or nonthermal inverse Compton emission. Depending on the uncertain efficiency of electron acceleration, this nonthermal emission could be more important than bremsstrahlung emission, particularly for small values of $\eta_{c}$.

Models in which thin accretion disks "evaporate" to form hot inner flows have received considerable attention in the context of dwarf novae (Meyer \& Meyer-Hofmeister 1994), soft X-ray transients in quiescence (Narayan, McClintock, \& Yi 1996; Honma 1996; Meyer, Liu, \& Meyer-Hofmeister 2000, Rozanska \& Czerny 2000), and low luminosity AGN (Lasota et al. 1996; Quataert et al. 1999). The energy for "evaporation" is ultimately thought to originate in the hot inner flow. Convection in CDAFs transports a significant amount of energy to large radii. CDAFs should thus be much more efficient than ADAFs at evaporating the outer disk. The implications of convective energy transport for theoretical models of the transition from thin disks to hot flows deserve further investigation.

Acknowledgment: The authors are grateful to an anonymous referee for a number of suggestions which helped improve the presentation. This work was supported in part by grants PHY 9507695 and AST 9820686 from the National Science Foundation. GB was supported by a Frank Knox Memorial Fellowship from Harvard University. EQ is supported by NASA through Chandra Fellowship PF9-10008, awarded by the Chandra X-ray Center, which is operated by the Smithsonian Astrophysical Observatory for NASA under contract NAS 8-39073 


\section{REFERENCES}

Abramowicz, M., Chen, X., Kato, S., Lasota, J.P., \& Regev, O., 1995, ApJ, 438, L37

Begelman, M.C. \& Chiueh, T., 1988, ApJ, 332, 872

Begelman, M.C. \& Meier, D.L., 1982, ApJ, 253, 873

Blandford, R.D. \& Begelman, M.C., 1999, MNRAS, 303, L1

Di Matteo, T., Fabian, A. C., Rees, M. J., Carilli, C. L., \& Ivison, R. J., 1999, MNRAS, 305, 492

Di Matteo, T., Quataert, E., Allen, S., Narayan, R., \& Fabian, A. C., 2000, MNRAS, 311, 507

Esin, A.A., McClintock, J.E., \& Narayan, R. 1997, ApJ, 489, 865

Fabian, A.C. \& Rees, M. J. 1995, MNRAS, 277, L5

Honma, F., 1996, PASJ, 48, 77

Ichimaru, 1977, ApJ, 214, 840

Igumenshchev, I.V., Chen, X., \& Abramowicz, M. A., 1996, MNRAS, 278, 236

Igumenshchev, I.V. \& Abramowicz, M. A., 1999, MNRAS, 303, 309

Igumenshchev, I.V. \& Abramowicz, M. A., 2000, ApJ, in press astro-ph/0003397)

Igumenshchev, I.V., Abramowicz, M. A., \& Narayan, R., 2000, ApJ, 537, L27

Kato, S., Fukue, U., \& Mineshige, S. 1998, Black Hole Accretion Disks (Kyoto: Kyoto Univ. Press)

Koyama, K., Maeda, Y., Sonobe, T., Takeshima, T., Tanaka, Y. \& Yamauchi, S., 1996, PASJ, 48, 249

Lasota, J.-P., Abramowicz, M.A., Chen, X., Krolik, J., Narayan, R., \& Yi, I., 1996, ApJ, 462, L142

Mahadevan, R. 1997, ApJ, 477, 585

Mahadevan, R. 1998, Nature, 394, 651

Mahadevan, R. \& Quataert, E. 1997, ApJ, 490, 605

Melia, F., 1992, ApJ, 387, L25

Meyer, F. \& Meyer-Hofmeister, E., 1994, A\&A, 288, 175

Meyer, F., Liu, B.F., \& Meyer-Hofmeister, E., 2000, A\&A, 354, L67

Narayan, R., Barret, D., \& McClintock, J. E., 1997a, ApJ, 482, 448

Narayan, R., Igumenshchev, I. V., \& Abramowicz, M. A., 2000, ApJ, 539, 798

Narayan, R., Mahadevan, R., \& Quataert, E. 1999, in The Theory of Black Hole Accretion Discs, ed. M. A. Abramowicz, G. Bjornsson, \& J. E. Pringle (Cambridge: Cambridge Univ. Press) p148

Narayan, R., McClintock, J.E., \& Yi, I., 1996, ApJ, 457821 
Narayan, R. \& Raymond, R., 1999, ApJ, 515, L69

Narayan, R. \& Yi, I., 1994, ApJ, 428, L13

Narayan, R. \& Yi, I., 1995, ApJ, 444, 231

Narayan, R., Yi, I., \& Mahadevan, R., 1995, Nature, 374, 623

Özel, F. \& Di Matteo, T., 2000, ApJ, in press astro-ph/0004267)

Özel, F., Psaltis, D., \& Narayan, R., 2000, ApJ, in press (astro-ph/0004195

Quataert, E. \& Narayan, R., 1999a, ApJ, 520, 298

Quataert, E. \& Narayan, R., 1999b, ApJ, 528, 236

Quataert, E. \& Narayan, R., 1999c, ApJ, 516, 399

Quataert, E. \& Gruzinov, A., 2000, ApJ, 539, 809 astro-ph/9912440

Quataert, E., Di Matteo, T., Narayan, R., \& Ho, L.C., 1999, ApJ, 525, L89

Rees, M. J., Phinney, E. S., Begelman, M. C. \& Blandford, R. D., 1982, Nature, 295, 17

Rozanska, A. \& Czerny, B. 2000, A\&A, 360, 1170

Ryu, D. \& Goodman, J., 1992, ApJ, 338, 438

Stone, J. M. \& Balbus, S. A., 1996, ApJ, 464, 364

Stone, J. M., Pringle, J. E., \& Begelman, M. C., 1999, MNRAS, 310, 1002 


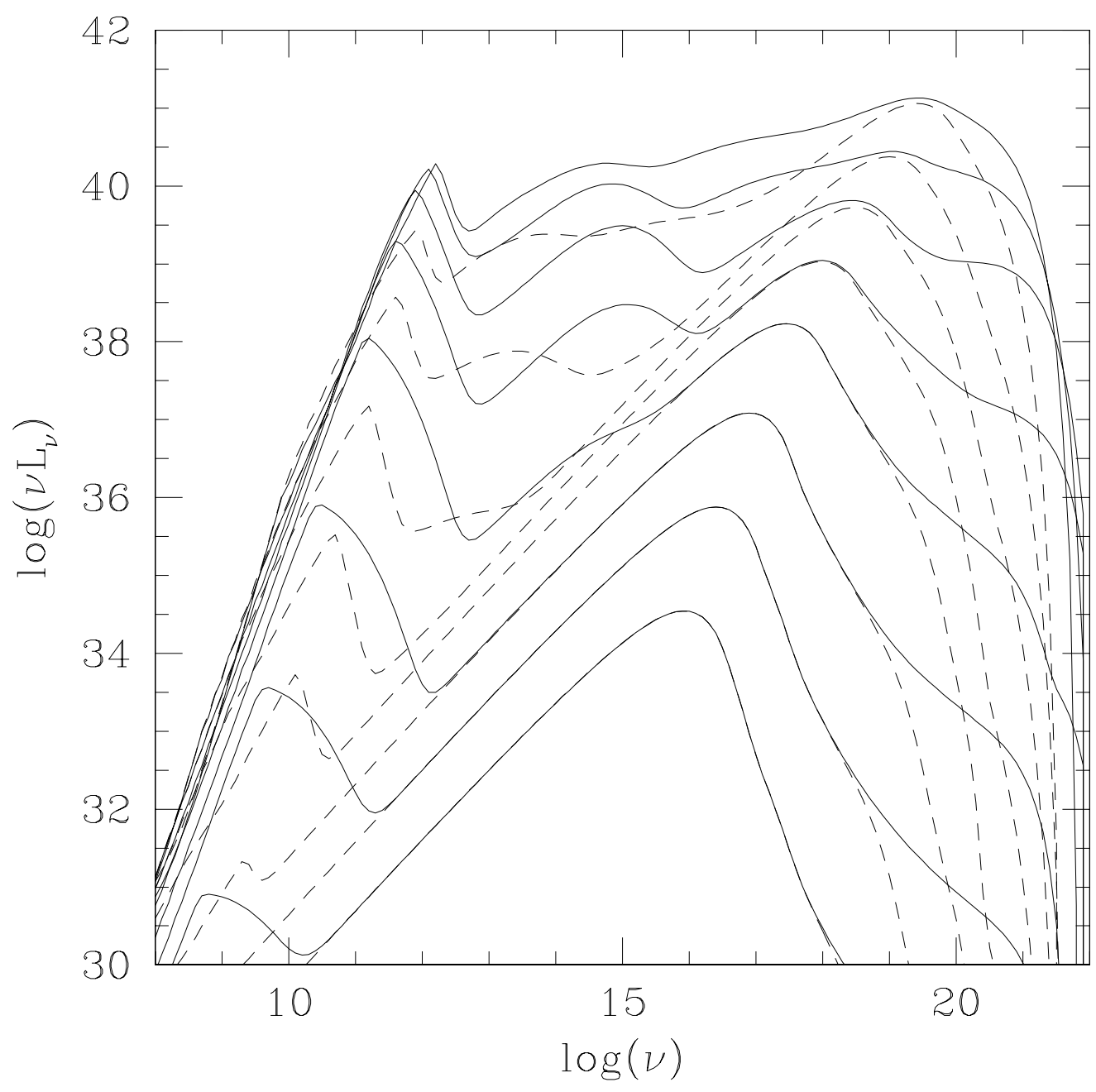

Fig. 1. - Model CDAF spectra for a $10^{8} M_{\odot}$ black hole, taking $\eta_{c}=1$. Dashed lines are for a fraction $\delta=0.01$ of the viscous energy heating the electrons. From top to bottom, the curves correspond to models with $\left(\log r_{\text {out }}, \log \dot{m}\right)=(2.5,-3.29),(3.0,-3.86),(3.5,-4.49),(4.0,-5.19),(4.5,-5.99),(5.0,-7.15),(5.5,-8.36)$, $(6.0,-9.70)$, respectively. Solid lines are for $\delta=0.5$. From top to bottom, the curves correspond to models with $\left(\log r_{\text {out }}, \log \dot{m}\right)=(2.5,-3.38),(3.0,-3.91),(3.5,-4.50),(4.0,-5.19),(4.5,-5.99),(5.0,-7.15)$, $(5.5,-8.36),(6.0,-9.70)$, respectively. 

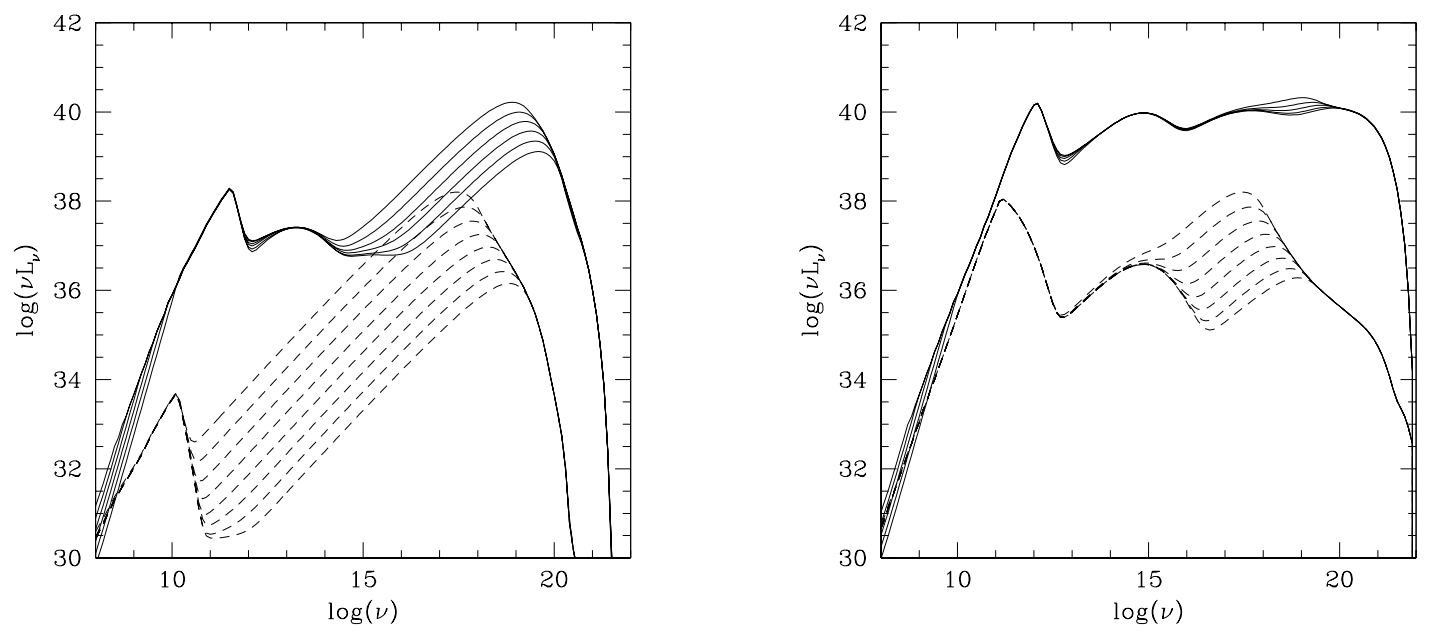

Fig. 2.- Model CDAF spectra for a $10^{8} M_{\odot}$ black hole. Left panel: Models with $\delta=0.01$. The solid curves correspond to $\dot{m}=10^{-4}$ and, from top to bottom, $\left(\log r_{\text {out }}, \eta_{c}\right)=(3.1,0.96),(2.9,0.55),(2.7,0.31)$, $(2.5,0.16),(2.3,0.074),(2.1,0.019)$, respectively. Dashed curves correspond to $\dot{m}=10^{-6}$ and, from top to bottom, $\left(\log r_{\text {out }}, \eta_{c}\right)=(4.5,0.97),(4.3,0.45),(4.1,0.22),(3.9,0.11),(3.7,0.056),(3.5,0.029),(3.3,0.015)$, $(3.1,0.0080)$, respectively. Right panel: Models with $\delta=0.5$. The solid curves correspond to $\dot{m}=10^{-4}$ and, from top to bottom, $\left(\log r_{\text {out }}, \eta_{c}\right)=(3.0,0.86),(2.8,0.54),(2.6,0.34),(2.4,0.20),(2.2,0.094)$, respectively. Dashed curves correspond to $\dot{m}=10^{-6}$ and, from top to bottom, $\left(\log r_{\text {out }}, \eta_{c}\right)=(4.5,0.97),(4.3,0.45)$, $(4.1,0.22),(3.9,0.11),(3.7,0.059),(3.5,0.032),(3.3,0.018),(3.1,0.011)$, respectively. 


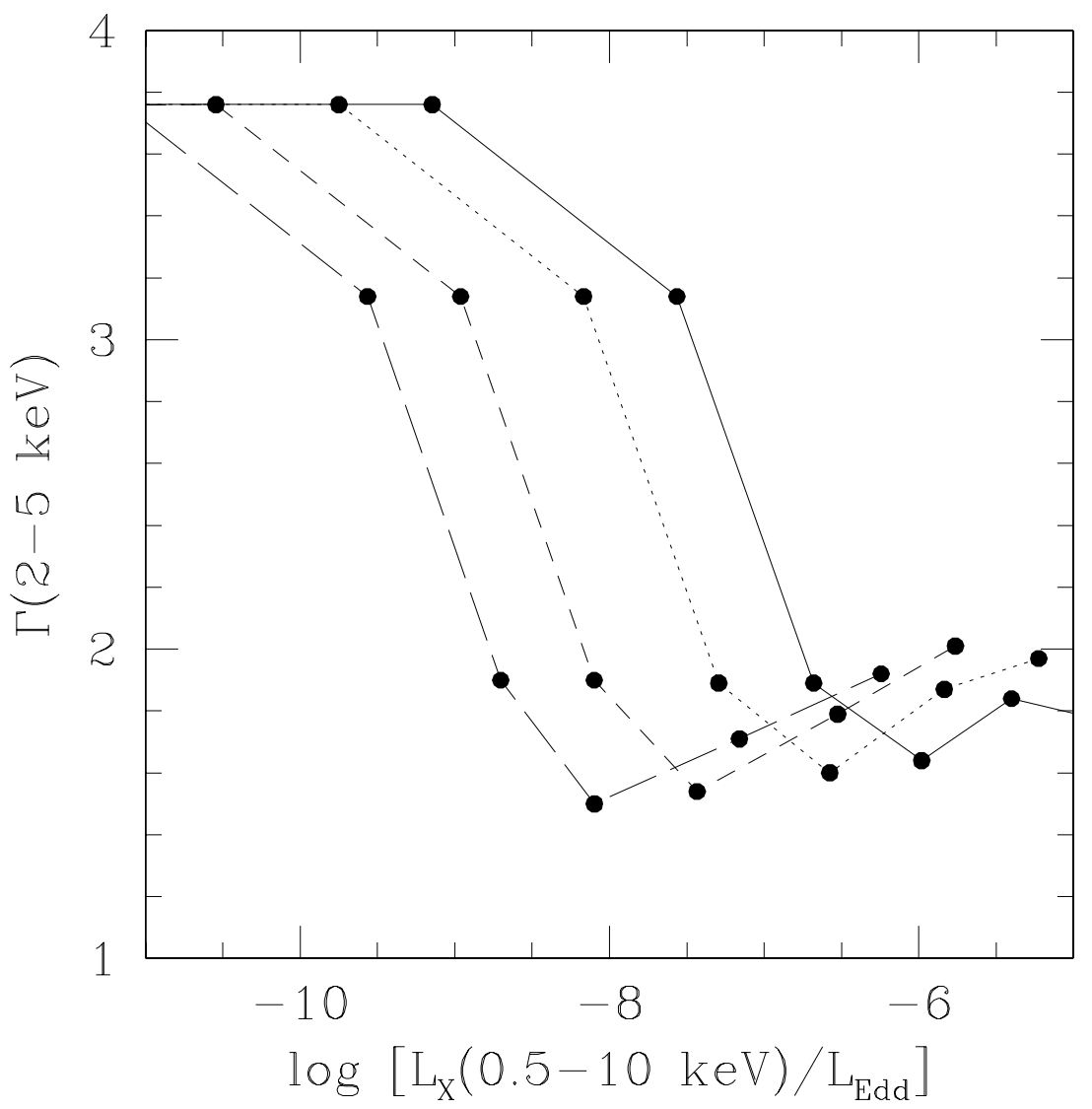

Fig. 3.- X-ray photon index vs. X-ray luminosity for models with $\delta=0.5$. Solid, dotted, dashed, and long-dashed curves correspond to $\eta_{c}=1,0.5,0.2$, and 0.1 , respectively. 

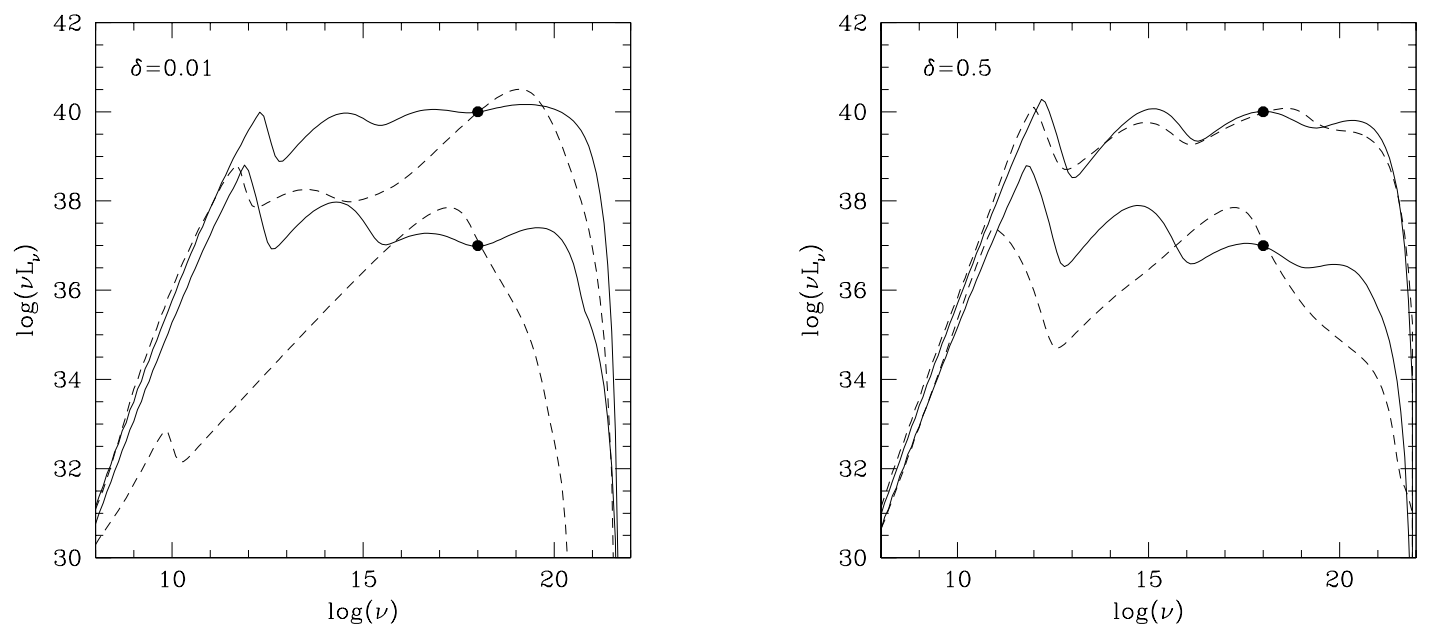

Fig. 4. - Comparison of ADAF and CDAF model spectra; $\eta_{c}=1$ for the CDAF models. The accretion rates in the models have been adjusted so that the $1 \mathrm{keV}$ luminosities are equal to either $10^{37} \mathrm{erg} \mathrm{s}^{-1}$ or $10^{40} \mathrm{erg} \mathrm{s}^{-1}$ (solid circles). Left panel: Models with $\delta=0.01$. The ADAF models (solid curves) correspond, from above, to $\left(\log r_{\text {out }}, \log \dot{m}\right)=(4.9,-2.74)$ and $(5.2,-3.64)$, respectively, and the CDAF models (dashed curves) correspond to $\left(\log r_{\text {out }}, \log \dot{m}\right)=(2.9,3.75)$ and $(4.7,-6.37)$, respectively. Right panel: Models with $\delta=0.5$. The ADAF models (solid curves) correspond to $\left(\log r_{\text {out }}, \log \dot{m}\right)=(5.5,-3.53)$ and $(5.6,-4.20)$, respectively, and the CDAF models (dashed curves) correspond to $\left(\log r_{\text {out }}, \log \dot{m}\right)=(3.3,-4.26)$ and $(4.7,-6.37)$, respectively. 